\title{
Global context effects on processing lexically ambiguous words: Evidence from eye fixations
}

\author{
GRETCHEN KAMBE, KEITH RAYNER, and SUSAN A. DUFFY \\ University of Massachusetts, Amherst, Massachusetts
}

\begin{abstract}
Readers' eye movements were monitored as they read biased ambiguous target words in the context of a short paragraph. Two aspects of context were manipulated. The global context was presented in the topic sentence of the paragraph and instantiated either the dominant or the subordinate meaning of biased ambiguous target words (those with highly dominant meanings). Local contextual information either preceded or followed the target word and was always consistent with the subordinate interpretation. Consistent with prior research, we obtained a subordinate bias effect wherein readers looked longer at the ambiguous words than control words when the preceding context instantiated the subordinate meaning. More importantly, the magnitude of the subordinate bias effect was the same when global context alone, local context alone, or local and global context combined were consistent with the subordinate meaning of the ambiguous word. The results of this study indicate that global contextual information (1) has an immediate impact on lexical ambiguity resolution when no local disambiguating information is available, (2) has no additional effect when it is consistent with local information, but (3) does have a slightly delayed effect when inconsistent with local information.
\end{abstract}

A great deal of research has made it clear that contextual information influences the processing of lexically ambiguous words (Binder \& Morris, 1995; Dopkins, Morris, \& Rayner, 1992; Duffy, Morris, \& Rayner, 1988; Kintsch \& Mross, 1985; Neill, 1989; Onifer \& Swinney, 1981; Rayner \& Duffy, 1986; Rayner \& Frazier, 1989; Rayner, Pacht, \& Duffy, 1994; Sereno, 1995; Sereno, Pacht, \& Rayner, 1992; Simpson, 1984; Swinney, 1979; Tanenhaus, Leiman, \& Seidenberg, 1979; Till, Mross, \& Kintsch, 1988). The majority of this research has been devoted to studies containing only one or two sentences, and there has been much less research on lexical ambiguity resolution within longer discourse passages. However, understanding how the information provided within a discourse context influences comprehension is important because sentences usually appear in a discourse context, not in isolation. During reading, a coherent discourse representation is formed that can affect the understanding and processing of successive sentences. It is the impact of

The data reported in this article are based on a master's thesis submitted by G.K. to the Graduate School of the University of Massachusetts. The research was supported by Grant HD17426 from the National Institutes of Health. G.K. was supported by a predoctoral traineeship on Grant MH16745 from the National Institute of Mental Health. K.R. was supported by a Research Scientist Award (MH01255) from the National Institute of Mental Health. We thank the other members of the master's committee (Nancy A. Myers and Sandy Pollatsek) and two reviewers (Peter Dixon and Matt Traxler) for their helpful comments. Portions of the data were presented at the 1999 meetings of the Eastern Psychological Association and the Midwestern Psychological Association. Correspondence should be addressed to K. Rayner, Department of Psychology, University of Massachusetts, Amherst, MA 01003 (e-mail: rayner@psych.umass.edu). this discourse-level representation on lexical ambiguity resolution that is addressed in this article.

Many researchers agree that there is more than one source of contextual influence on the processing of a target word (Binder \& Morris, 1995; Hess, Foss, \& Carroll, 1995; Kintsch \& Mross, 1985; Morris, 1994; Rayner et al., 1994; Schustack, Ehrlich, \& Rayner, 1987; Schwanenflugel \& White, 1991; Simpson, 1984; Till et al., 1988). Two major sources of information are (1) the topic of the paragraph and (2) the information immediately preceding the target word in the current sentence. The topic is usually instantiated in the first sentence of a paragraph and provides the reader with a general idea of what is going to be discussed; we will refer to this as the global or discourse context of the passage. We will refer to the information contained within the current sentence as the local context; the local context usually provides information specific to the ideas being discussed in the paragraph.

In the present experiment, we examined the effects of global and local context on the processing of lexically ambiguous words by recording readers' eye movements as they read short passages of text in which we manipulated these two types of context. Several recent eye movement studies (Binder \& Morris, 1995; Dopkins et al., 1992; Duffy et al., 1988; Rayner \& Duffy, 1986; Rayner \& Frazier, 1989; Rayner et al., 1994; Sereno, 1995; Sereno et al., 1992) examined readers' fixation times on ambiguous words and control words (matched in length and frequency). Two interesting findings have emerged from this research that have been interpreted within the context of the reordered access model (Duffy et al., 1988). First, when the preceding context was neutral, readers fixated longer on balanced ambiguous words (with two equally likely meanings) than on either biased ambiguous 
words (with a highly dominant meaning) or unambiguous control words; no difference in fixation times was found between biased ambiguous words and controls. However, significantly more time was spent on the disambiguating posttarget region in the sentences when the biased ambiguous words were consistent with the subordinate meaning of the word. This suggests that the reader originally interpreted the biased ambiguous word in accordance with its dominant meaning. This makes sense if the dominant interpretation is available prior to the subordinate meaning of a biased ambiguous word in neutral sentence contexts. The reader would have accepted the first available meaning of the biased ambiguous target word (the dominant meaning) and integrated it into the ongoing sentence context. This would have taken the same amount of time as processing an unambiguous word as both would be initially interpreted as consisting of a single meaning. Second, and more interestingly, when disambiguating information preceded the ambiguous word and instantiated the subordinate meaning, fixation times on biased ambiguous words were longer than on balanced ambiguous words, unambiguous control words, or biased ambiguous words that were disambiguated toward the dominant meaning. This slowdown when the subordinate meaning is instantiated by prior context is referred to as the $s u b$ ordinate bias effect (Pacht \& Rayner, 1993; Rayner et al., 1994). Under the assumptions of the reordered access model, when disambiguating information related to the subordinate meaning of an ambiguous word is available in the preceding local context, the speed of activation for the subordinate meaning is increased. Thus, the subordinate meaning becomes available in the same window of time as the dominant meaning. The resulting competition between the subordinate and dominant meaning of the word increases processing time for the target word, producing the subordinate bias effect.

Rayner et al. (1994) conducted two studies to determine whether a stronger prior context would reduce or eliminate the subordinate bias effect. They reasoned that a prior encounter with the subordinate meaning of a biased ambiguous word would increase the availability of the subordinate meaning upon second encounter of the same word (when the subordinate meaning was again instantiated). This increase in availability might allow the subordinate meaning to be accessed earlier than the dominant meaning. In two studies Rayner et al. failed to eliminate or reduce the subordinate bias effect. Rephrased in terms of global and local context, in these studies the subordinate bias effect induced by local context was not modified by the addition of global context that also supported the subordinate meaning (see also Binder \& Rayner, 1998). ${ }^{1}$ This suggests that two sources of contextual support for the subordinate meaning (global context and local context) do not sum (to create twice as much support as only one source); rather, they simply provide one source of support. This is reasonable if one views the local context as simply providing a continuation of the global context. Support for this view is provided in a series of stud- ies by Hess et al. (1995) in which they found evidence for an overall effect of global context on the processing of unambiguous words rather than separate effects of global and local context.

In the experiment reported here, we utilized the subordinate bias effect to examine the effect of global and local context on the processing of biased ambiguous words. Readers' eye movements were monitored as they read short passages of text containing biased ambiguous target words (or unambiguous control words). Disambiguating information was provided in the local context, the global context, or both. The subordinate meaning of the ambiguous target words was always ultimately instantiated by local disambiguating information that either preceded or followed the ambiguous target word (the local-before and the local-after conditions). Global context was always presented in the first sentence of the passage and was consistent with either the dominant or subordinate meaning of the ambiguous word (globaldominant and global-subordinate conditions). The resulting combination of conditions yielded four versions (Appendix A) of each paragraph context: global dominant-local before, global dominant-local after, global subordinate-local before, and global subordinate-local after. Note that for all conditions except the global dominant-local after, preceding context provides information supporting the (intended) subordinate meaning of the ambiguous word. In the global subordinate-local after condition, support for the subordinate meaning comes only from the global context. In the global dominantlocal before condition, support for the subordinate meaning comes only from the local context. In the global subordinate-local before condition, support for the subordinate meaning comes from both local and global context (similar to Rayner et al., 1994).

To assess the individual contribution of the global and local contexts, fixation times were examined in three critical regions varying in size: the target region, the posttarget region, and the disambiguating. The target region consisted of a biased ambiguous word or an unambiguous control word matched on length and frequency. ${ }^{2}$ The posttarget region began immediately after the target word and consisted of two to five words. The posttarget region was neutral with respect to the meaning of the target word. The disambiguating region either (1) began the target sentence and ended at the target word or (2) began at the end of the posttarget region and extended to the end of the target sentence. When the disambiguating information came after the target word, the beginning of the sentence contained neutral information. When the disambiguating information preceded the target word, the end of the sentence consisted of a logical continuation of the sentence.

Different predictions can be made with respect to the impact that global and local contexts have on the subordinate bias effect for target words. ${ }^{3}$ Consistent with the results of Rayner et al. (1994) and Hess et al. (1995) would be a finding of similar effects for all three condi- 
tions in which the prior context has some information supporting the subordinate meaning (whether it be the global context, the local context,or both). Alternatively, the two sources of contextual constraint might operate independently. In this case, in the global subordinate-local before condition the two sources of constraint should combine to produce stronger support for the subordinate meaning. This should reduce the subordinate bias effect for this condition because there will be more trials on which the subordinate meaning is actually activated earlier than the dominant meaning (resulting in no conflict between the two meanings). A final possibility is that not only are local and global contexts independent sources of constraint, but because the global context is more distant, its effect is delayed. Thus, the subordinate bias effect on the target word itself would reflect for the most part the effect of local context; a subordinate bias effect should emerge in the global subordinate-local before and the global dominant-local before conditions but not in the global subordinate-local after condition. A delayed effect of global context for the global subordinatelocal after condition might then be observed in the posttarget regions.

Finally, it should be noted that all theoretical accounts predict no subordinate bias effect for the global dominantlocal after condition in which the preceding context supports the dominant meaning of the ambiguous word. In this case, the dominant meaning should become available earlier than the subordinate meaning and will be easily integrated into the discourse. Processing difficulty should emerge, however, when the reader finally encounters the local context information later in the sentence. At this point the reader will have to engage in a timeconsuming process of reprocessing the ambiguous word and finding its subordinate meaning.

\section{METHOD}

\section{Participants}

Forty-eight members of the University of Massachusetts community received payment or course credit for their participation in the study. They were all native English speakers with normal or corrected vision and were naive with respect to the purpose of the study.

\section{Apparatus}

Eye movements were recorded by a Fourward Technologies Dual Purkinje Eyetracker (Generation V), which has a resolution of less than $10^{\prime}$ of arc. The participants' view of the screen was binocular, but only the right eye was monitored for eye location. The signal from the eyetracker was sampled every millisecond by a 486 computer. The passages were double-spaced and presented on an NEC MultiSync 4FG color monitor that was interfaced with the computer. The participants were seated $60 \mathrm{~cm}$ from the screen with three characters equaling $1^{\circ}$ of visual angle. The luminance of the screen was adjusted to a comfortable level of brightness for the participant and then held constant throughout the study. The experimental room was dark except for a small indirect light that enabled the experimenter to keep notes during the experiment.

\section{Procedure}

When participants arrived for the experiment, they were told that they would be expected to read passages on a computer screen while their eye movements were monitored. They were also told that they would occasionally be asked comprehension questions about the passage they read. All comprehension questions required a simple yes/no response made by pushing a button. Approximately $25 \%$ of the passages were followed by a question. After the procedure was explained, a bite bar (used to eliminate head movements) was prepared for each participant.

Once the participant was seated in front of the monitor, an initial calibration procedure that took approximately 5 min was completed. The calibration of the eyetracking system was checked between each passage to ensure that accurate records were being obtained. When the experimenter determined that the participant was accurately calibrated and fixating on the upper left part of the screen, the entire passage was presented. The participant was instructed to push a button to erase the passage from the screen when he/she had completed reading it. Once the passage had been erased and the trial ended, the calibration pattern reappeared and the next passage was presented. This procedure was repeated throughout the entire practice and experimental sessions.

\section{Materials}

Twenty-four biased ambiguous words were chosen from a series of norms collected at the University of Massachusetts, as well as published norms (Twilley, Dixon, Taylor, \& Clark, 1994). The dominant sense of these biased words had a probability range of .83-.99 with a mean of .90 . The subordinate sense had a probability range of .01-.14 with a mean of .06. Each ambiguous word was paired with an unambiguous control word that was matched in length of letters and frequency of occurrence in accordance with the Francis and Kučera (1982) norms (see Appendix B). Control words were selected to be consistent with the subordinate sense of the ambiguous target word and the local contextual information. The average word frequency count for the biased ambiguous words was 80.6 (range $=$ $1-361$ ) and 80.5 (range $=3-342$ ) for their matched control words.

For each word pair, two different passages were constructed in which each member of the word pair fit smoothly into the passage. Each passage contained a topic sentence, two filler sentences, and a target sentence. The topic sentence introduced the global context, which was related to either the subordinate or dominant meaning of the ambiguous word. The target sentence contained the local context and was always biased toward the subordinate meaning of the ambiguous target word. This local disambiguating information either preceded or followed the target word.

The topic of the discourse passage was introduced in the first sentence and established a situation that was consistent either with the subordinate meaning of the ambiguous word (global subordinate) or with the dominant meaning of the ambiguous word (global dominant). For example, in the global subordinate conditions (Appendix A) the topic sentence for the ambiguous word band was "Lisa and John spent months looking for the perfect wedding ring," which was consistent with the less frequent jewelry meaning of band. The topic sentence for the global-dominant condition was "Lisa and John loved to go to rock concerts with their friends," which was consistent with the more frequent music meaning of band. Each topic sentence was followed by two neutral filler sentences (Appendix A). The same two filler sentences were used across all passages. The filler sentences were congruous continuations of the initial sentence (regardless of the topic condition) and were neutral with respect to the different meanings of the ambiguous words.

The target sentence of each passage contained the local context and was semantically biased toward the subordinate meaning of the ambiguous target word. For example, in a passage in which band 
was the ambiguous word, the local context was biased toward the jewelry meaning of the word. This disambiguating information in the local context either preceded (local before) or followed (local after) the target word. When it preceded the target word, information related to the subordinate meaning of the ambiguous word (such as "jewelry store") was available immediately prior to encountering the target word. The sentence "It wasn't until they entered Kay's Jewelry store that they saw the band ..." (Appendix A) is an example of the conditions in which the local contextual information was available before the target word was encountered. When local disambiguating information was not available until after the ambiguous word was encountered, the context immediately preceding the target word was neutral with respect to the meaning of the ambiguous word. The sentence "One day they decided to go to New York City to see the band ..." (Appendix A) is an example of the condition where the local context was available after the ambiguous word was encountered. Two posttarget regions followed the target word. The first consisted of two to five words and was neutral with respect to either meaning of the ambiguous word; the second contained either the disambiguating information (in the local-after conditions) or a natural continuation of the sentence (in the local-before conditions). The neutral posttarget region was critical in the local after condition because it allowed us to observe whether there was a delayed effect of global context before the local disambiguating information was encountered.

There were a total of eight within-subjects conditions formed by crossing three factors: global context (subordinate vs. dominant), local context (before vs. after the target word), and ambiguity (ambiguous vs. control). All eight factors were counterbalanced using a Latin square design, and the order of presentation was always randomized. Each participant read 48 experimental passages, six passages in each of the eight conditions, and 48 filler passages. They saw every ambiguous word as well as its matched control word, although the ambiguous words and their matched controls were always presented in different passage frames. So, if a participant saw the ambiguous target word band in the rock concert passage, he/she would encounter the control word gold in the other passage frame (the rock star interview paragraph). As a result, no target word, control word, or passage frame was ever repeated for a particular participant. Example paragraphs are presented in Appendix A.

\section{RESULTS AND DISCUSSION}

Processing time measures were calculated for three regions within the target sentence: the target word, the posttarget region (consisting of two to five words that were identical across conditions), and the disambiguating region (which averaged about 12 words). Analyses of variance (ANOVAs) were performed on these data using both

Table 1

Gaze Duration (GD), First Fixation Duration (FFD), and Total Time (TT, in Milliseconds) on the Target Word

\begin{tabular}{lrrrrrr}
\hline & \multicolumn{3}{c}{ Local Before } & & \multicolumn{3}{c}{ Local After } \\
\cline { 2 - 5 } \cline { 5 - 7 } Target & GD & FFD & TT & GD & FFD & TT \\
\hline Ambiguous & 303 & 283 & 344 & 304 & 283 & 353 \\
Control & 286 & 276 & 305 & 284 & 264 & 327 \\
Difference & 17 & 7 & 39 & 20 & 19 & 26 \\
\multicolumn{7}{c}{ Global-Dominant Context } \\
Ambiguous & 304 & 284 & 372 & 298 & 276 & 354 \\
Control & 291 & 268 & 330 & 297 & 281 & 356 \\
Difference & 13 & 16 & 42 & 1 & -5 & -2 \\
\hline
\end{tabular}

participant $\left(F_{1}\right)$ and item $\left(F_{2}\right)$ variability. Unless otherwise noted, all $p$ s are $<.05$.

The primary processing time measure that was calculated for the target word was the gaze duration, but we also analyzed the first fixation duration and the total fixation time on the target word. Gaze duration is conditional on the word being fixated with a forward fixation and was calculated by summing all consecutive fixations on a word before leaving that word. Thus, this measure consists of the processing time involved in the reader's initial encounter of the target word, and does not include any regressions from other regions of the text. If a target word was not actually fixated, the closest fixation within three character spaces to the left and one to the right, was counted as the fixation during which the target word was processed (Rayner \& Pollatsek, 1989). Like gaze duration, first fixation duration is conditional on the word being fixated on a forward fixation, but consists of the duration of the first fixation on a word regardless of how many additional times the reader fixated on the word. While in many contexts this measure has provided valuable information, in most studies dealing with the processing of lexically ambiguous words, the gaze duration has turned out to be the most informative measure. The total time measure includes all fixations on the target word, including regressions back to it. Fixations less than $100 \mathrm{msec}$ were eliminated since such short fixation times are assumed to reflect oculomotor programming or processing that actually took place during the prior fixation (Morrison, 1984). Additionally, fixation times longer than $1,000 \mathrm{msec}$ were assumed to reflect track losses or eye blinks, and were thus eliminated. This resulted in $7 \%$ of the data being excluded from the analyses. For regions other than the target word, first-pass reading time, total reading time, and go-past reading time measures are reported. These measures are standard (Rayner, 1998) for regions larger than a single word. First-pass time is equivalent to the gaze duration measure on a single target word. The go-past measure is described in more detail later.

\section{Target Word}

Table 1 shows the gaze duration, first fixation duration, and total time on the target word for the conditions in the experiment. Separate 2 (global context: subordinate vs. dominant interpretation) $\times 2$ (local context: before vs. after) $\times 2$ (ambiguity: ambiguous vs. control) ANOVAs were performed on the data. Across the measures, there was a clear main effect of ambiguity since fixation times were longer on the ambiguous target word than on the unambiguous control word: for gaze duration ${ }^{4}$ $\left[F_{1}(1,47)=5.07, M S_{\mathrm{e}}=2,997, F_{2}(1,47)=5.55, M S_{\mathrm{e}}=\right.$ $5,918]$, first fixation duration $\left[F_{1}(1,47)=5.36, M S_{\mathrm{e}}=\right.$ $\left.1,469, F_{2}(1,47)=4.02, M S_{\mathrm{e}}=2,192\right]$, and total time $\left[F_{1}(1,47)=7.43, M S_{\mathrm{e}}=5,788, F_{2}(1,47)=5.26, M S_{\mathrm{e}}=\right.$ $12,497]$. Two subsequent analyses were performed. The first tested whether the subordinate bias effect differed in magnitude for the three conditions in which it could oc- 
cur (global subordinate-local before, global subordinatelocal after, and global dominant-local before). The second established that there was no subordinate bias effect in the global dominant-local after condition. First, an ANOVA on the gaze durations that included the global subordinate-local before, global subordinate-local after, and global dominant-local before conditions confirmed that readers spent more time on the ambiguous target word compared with the control word as indicated by the significant effect of ambiguity $\left[F_{1}(1,47)=5.39, M S_{\mathrm{e}}=1,495\right.$, $\left.F_{2}(1,47)=4.77, M S_{\mathrm{e}}=4,234\right] .{ }^{5}$ No other effects were significant. Second, for the global dominant-local after condition, the gaze duration means for the ambiguous target word and the control word did not differ (all $F$ s $<1){ }^{6}$

For gaze duration and first fixation duration, no other main effects or interactions were significant. For total time, there was an additional main effect of global context $\left[F_{1}(1,47)=13.32, M S_{\mathrm{e}}=4,476, F_{2}(1,47)=7.12\right.$, $\left.M S_{\mathrm{e}}=5,784\right]$, wherein fixation times on the target word were longer in the global-dominant conditions $(353 \mathrm{msec})$ than in the global-subordinate conditions $(332 \mathrm{msec})$. Subsequent analyses suggested that this effect was a second-pass effect of the global context. Second-pass reading times for the target word were longer when the global context was biased toward the dominant meaning of the ambiguous target word as revealed by a main effect of global context $\left[F_{1}(1,47)=9.15, M S_{\mathrm{e}}=4,063\right.$, $\left.F_{2}(1,47)=14.54, M S_{\mathrm{e}}=2,692\right]$.

Fixation times on the target word revealed that readers spent more time on a subordinately biased ambiguous word than a control word matched on length and frequency. What is significant about the current data is that the subordinately biased contextual information did not have to be immediately prior to the ambiguous target word. In fact, the subordinately biased information in the global subordinate-local after condition had an immediate impact on lexical ambiguity resolution in the absence of immediately preceding sentential context, even though the subordinate information only appeared in the topic sentence of the paragraph. Interestingly, the subordinate bias effect was not affected by the amount of subordinately biased information available, or the distance of that information to the target word. For example, the magnitude of the subordinate bias effect was equivalent for the global subordinate-local before condition (where there were two sources of biasing information), the global subordinate-local after condition (where a single distant source of biasing information was available), and the global dominant-local before condition (where a single close source of biasing information was available). However, while there was no effect of global context in first fixation and gaze duration for the global dominant-local before condition, total time measures revealed that readers did spend more time on the target word when the global contextual bias was inconsistent with the local-before contextual bias. Presumably, the change in topic that occurred between the global (dominant) and local (subordinate) context increased the difficulty of comprehension
Table 2

First Fixation Duration (FFD) $(n+1)$ and Go-Past (GP, in Milliseconds) on the Spillover and Posttarget Regions

\begin{tabular}{|c|c|c|c|c|}
\hline \multirow[b]{2}{*}{ Target } & \multicolumn{2}{|c|}{ Local Before } & \multicolumn{2}{|c|}{ Local After } \\
\hline & FFD & GP & FFD & GP \\
\hline \multicolumn{5}{|c|}{ Global-Subordinate Context } \\
\hline Ambiguous & 260 & 474 & 265 & 487 \\
\hline Control & 268 & 455 & 258 & 477 \\
\hline Difference & -8 & 19 & 7 & 10 \\
\hline \multicolumn{5}{|c|}{ Global-Dominant Context } \\
\hline Ambiguous & 289 & 520 & 267 & 470 \\
\hline Control & 270 & 472 & 275 & 523 \\
\hline Difference & 19 & 48 & -8 & -53 \\
\hline
\end{tabular}

processes for the text. As a result, the reader required additional time in second pass on the target word whether it was an ambiguous word or control word.

\section{Spillover Effects}

Table 2 shows the pattern of spillover effects (the duration of the first fixation immediately following firstpass fixations on the target word). This measure indicates whether the reader is continuing to encounter processing difficulty immediately after the target word. Here, a somewhat different pattern from the target word reading time is apparent. First, there was no main effect of ambiguity (all $F_{\mathrm{S}}<1$ ). There was, however, a significant main effect of global context, since the means for the context biasing toward the dominant meaning were longer than those when the context was biased toward the subordinate meaning $\left[F_{1}(1,47)=8.54, M S_{\mathrm{e}}=2,272\right.$, $\left.F_{2}(1,47)=6.60, M S_{\mathrm{e}}=2,175\right]$. The size of the effect varied as, indicated by a three-way interaction between global context, local context, and ambiguity $\left[F_{1}(1,47)=\right.$ $\left.4.04, M S_{\mathrm{e}}=2,214, F_{2}(1,47)=5.57, M S_{\mathrm{e}}=1,858\right]$. Readers' spillover fixations were longer for the ambiguous word than for the control word in the global dominantlocal before condition.

The change in topic between the global and local context for the global dominant-local before condition caused some processing difficulty for the ambiguous word. This increase in processing time is not surprising in light of the conflicting information that precedes the ambiguous target word in the global dominant-local before condition. The conflicting information requires readers to take more time to ensure that they are incorporating the appropriate meaning of the ambiguous target word into the ongoing discourse representation.

\section{Posttarget Region}

The posttarget region began immediately after the target word and consisted of two to five words that were identical across all passage conditions. This region separated the target word from the disambiguating information that followed (in the conditions where the local disambiguating context followed the target word) so spillover processing from the target word could be measured independent of processing of the disambiguating informa- 
Table 3

First-Pass (FP), Total Time (TT), and Go-Past (GP, in Milliseconds) on the Disambiguating Regions

\begin{tabular}{lccccrr}
\hline & \multicolumn{3}{c}{ Local Before } & & \multicolumn{2}{c}{ Local After } \\
\cline { 2 - 3 } Target & FP & TT & GP & & FP & TT \\
\hline Ambiguous & 1,908 & 2,212 & 2,000 & & 1,916 & 2,336 \\
Control & 1,906 & 2,134 & 2,013 & & 1,874 & 2,236 \\
Difference & 2 & 78 & -13 & & 42 & 100 \\
\multicolumn{7}{c}{ Global-Subordinate Context } \\
Ambiguous & 1,983 & 2,353 & 2,134 & & 2,122 & 2,645 \\
Control & 1,950 & 2,231 & 2,055 & & 1,910 & 2,355 \\
Difference & 33 & 122 & 79 & 212 & 290 \\
\hline
\end{tabular}

tion. In contrast to spillover, this measure indicates whether any unresolved processing difficulty from the target region is having an ongoing effect on processing. A go-past measure was used to analyze the data because this was a larger region within which readers occasionally made short regressions. The advantage of the particular go-past measure used is that it includes only time spent in the posttarget region (including regressions within the region), and it does not include time spent in the region once the reader has moved past it (to the right). Furthermore, it does not include any time spent outside of the posttarget region during regressions to prior portions of the text.

Time spent on the posttarget region is presented in Table 2. An ANOVA revealed an effect of global context that was significant by participants $\left[F_{1}(1,47)=5.94\right.$, $\left.M S_{\mathrm{e}}=22,140\right]$, but marginal by items $\left[F_{2}(1,47)=3.38\right.$, $\left.M S_{\mathrm{e}}=14,736, p<.08\right]$; more time was spent in this region when the global context was biased toward the dominant meaning of the ambiguous target word. In addition, an interaction between local context and ambiguity $\left[F_{1}(1,47)=8.24, M S_{\mathrm{e}}=13,734, F_{2}(1,47)=5.01\right.$, $\left.M S_{\mathrm{e}}=73,312\right]$ indicated that readers spent more time in this region if the local disambiguating information came before the target word and the target word was ambiguous. These effects were qualified by a significant threeway interaction between global context, local context, and ambiguity reflecting the finding that readers spent more time in the posttarget region after an ambiguous word for the first three conditions (global subordinatelocal before, global subordinate-local after, and global dominant-local before), but they spent more time in the posttarget region after the control word in the global dominant-local after condition $\left[F_{1}(1,47)=4.00, M S_{\mathrm{e}}=\right.$ $\left.9,865, F_{2}(1,47)=4.37, M S_{\mathrm{e}}=11,532\right]$. It is possible, however, that this interaction reflects difficulty in comprehending the control word in the global dominantlocal after condition rather than any interesting effect of ambiguity in the other conditions. For example, the reader might have slowed down while trying to determine how the unambiguous control word (i.e., gold) fit into the global-dominantcontext (i.e., rock concerts) in the global dominant-local after condition. Thus, an additional analysis was performed on the posttarget region for only the ambiguous word conditions to determine whether the increased reading time $(520 \mathrm{msec})$ after the ambiguous target word in the global dominant-local before condition differed from the others. An ANOVA performed on the posttarget region for the conditions that were preceded by an ambiguous target word yielded a significant interaction between the global and local contexts $\left[F_{1}(1,47)=\right.$ $\left.5.11, M S_{\mathrm{e}}=53,841, F_{2}(1,47)=6.13, M S_{\mathrm{e}}=38,299\right]$, indicating that readers spent more time in the posttarget region when the global context was biased toward the dominant meaning and the local context preceded an ambiguous target word. When the same analysis was performed on the posttarget region for the four conditions preceded by a control word, the interaction was not significant $\left(F_{\mathrm{S}}<1\right)$.

The posttarget region results are consistent with the spillover effects. Thus, readers encountered more processing difficulty after encountering an ambiguous target word in the global dominant-local before condition than in any other condition in which an ambiguous word was encountered. Readers spent the greatest amount of time in the posttarget region when the preceding global and local contexts instantiated different interpretations of the ambiguous target word.

\section{Disambiguating Region}

The disambiguating region either preceded or followed the target word. In the local-before conditions, the disambiguating information preceded the target word: The disambiguating information began the sentence and ended with the target word. In the local-after conditions, the disambiguating region followed the target word: The disambiguating information began immediately after the posttarget region and continued to the end of the sentence. As seen in Appendix A, the disambiguating information in the local-before conditions was lexically different from that in the local-after conditions, although the overall length of the regions was approximately the same (within $5 \%$ ). As a result, the disambiguating regions for the before and after conditions were analyzed separately. The contexts surrounding the target word were identical in both of the local-before conditions and also identical in both of the local-after conditions.

Time spent in the local-before disambiguating region is presented in Table 3 (mean first-pass time and mean total time). In the local-before conditions, there were no significant first-pass effects (all $F_{\mathrm{S}}<1$ ). Total time measures, however, revealed a significant main effect of global context $\left[F_{1}(1,47)=9.45, M S_{\mathrm{e}}=125,111, F_{2}(1,47)=\right.$ $\left.13.18, M S_{\mathrm{e}}=97,472\right]$. Readers spent more time in the disambiguating region when the global context was biased toward the dominant meaning of the ambiguous word. This result makes sense because the local disambiguating information was always biased toward the subordinate meaning of the ambiguous word and thus represented a shift in topic. In the global dominant-local before condition, this topic shift occurred immediately before encountering the target word. To determine whether 
the topic shift was noticed prior to reading the target word, a post hoc analysis was performed on the data using the go-past measure described above (Table 3). It is possible that the significant effect of global context in the total time measure may reflect rereading times after having gone past the disambiguating region and encountering the ambiguous target word. This was not the case. Readers spent more time in the disambiguating region when the global context was biased toward the dominant interpretation of the ambiguous word. An ANOVA on the go-past times for the local-before disambiguating region (the region up to but not including the target word) supported this finding $\left[F_{1}(1,47)=4.14, M S_{\mathrm{e}}=82,504\right.$, $\left.F_{2}(1,47)=5.21, M S_{\mathrm{e}}=70,956\right]$. This result indicates that the reader became aware of the context change before encountering the target word.

Time spent in the local-after disambiguating region is presented in Table 3 (first-pass time and total time). Firstpass effects revealed that readers spent more time in the local-after disambiguating region when it followed an ambiguous word than when it followed a control word $\left[F_{1}(1,47)=6.14, M S_{\mathrm{e}}=804,608, F_{2}(1,47)=5.09\right.$, $\left.M S_{\mathrm{e}}=151,210\right]$. Readers were also more likely to spend additional time in the disambiguating region when the global context was biased toward the dominant meaning of the ambiguous target word. This effect was significant by participants $\left[F_{1}(1,47)=5.35, M S_{\mathrm{e}}=136,321\right]$, but only marginally significant by items $\left[F_{2}(1,47)=3.32\right.$, $\left.M S_{\mathrm{e}}=210,697, p<.08\right]$. The effect of ambiguity was larger for the global-dominant condition, as indicated by an interaction between global context and ambiguity that was significant by participants, but not items $\left[F_{1}(1,47)=\right.$ $5.61, M S_{\mathrm{e}}=105,013, F_{2}(1,47)=1.79, M S_{\mathrm{e}}=194,579$, $p<.2]$.

Total time effects also revealed a significant main effect of ambiguity $\left[F_{1}(1,47)=23.13, M S_{\mathrm{e}}=88,091\right.$, $\left.F_{2}(1,47)=15.44, M S_{\mathrm{e}}=118,528\right]$; readers spent more time in the local-after disambiguating region when it followed an ambiguous word than when it followed a control word. In addition, readers were significantly more likely to spend extra time in the disambiguating region when the global context was biased toward the dominant meaning of the ambiguous word than when the global context was biased toward the subordinate meaning $\left[F_{1}(1,47)=31.60, M S_{\mathrm{e}}=68,948, F_{2}(1,47)=17.86\right.$, $\left.M S_{\mathrm{e}}=123,253\right]$. The effect of ambiguity was larger when the global context was biased toward the dominant rather than the subordinate meaning of the ambiguous target word. A significant interaction between global context and ambiguity supported this finding $\left[F_{1}(1,47)=8.25\right.$, $\left.M S_{\mathrm{e}}=60,784, F_{2}(1,47)=4.13, M S_{\mathrm{e}}=104,058\right]$.

In the global-dominant conditions, readers spent more time on the disambiguating region in the local-after condition following an ambiguous word than following its corresponding control word. Up to this point in the paragraph, there was no indication that the dominant interpretation of the ambiguous word was incorrect. Presumably, the reader had already completed meaning selection and had integrated the dominant meaning of the target word into the ongoing discourse representation. As a result, a time-consuming reanalysis was required once the discrepant disambiguating information was encountered. Not surprisingly, this effect in the local-after disambiguating region was not observed in the global-subordinate conditions. In these conditions, global context does allow the reader to select the subordinate meaning before encountering the local disambiguating region.

\section{DISCUSSION}

The purpose of the present experiment was to examine global and local context effects on lexical ambiguity resolution. Our results replicate previous studies of the subordinate bias effect (Binder \& Morris, 1995; Binder \& Rayner, 1998; Duffy et al., 1988; Rayner \& Frazier, 1989; Rayner et al., 1994) in that gaze durations were longer on biased ambiguous words in which the preceding context supported the subordinate meaning than on unambiguous control words. Longer gaze durations are consistent with the reordered access model and reflect processing difficulty due to accessing multiple meanings of the ambiguous target word. Presumably, the subordinate bias effect occurs because contextual information supporting the subordinate meaning speeds access for the less dominant interpretation of the ambiguous target word so that it becomes available in the same time window as the dominant meaning. This lengthens the time spent in selecting the intended meaning. The pattern of results demonstrates, consistent with the reordered access model, that the order in which the meanings of an ambiguous word become available is influenced by the prior context.

A more important finding from the present study is that the ambiguous target word was read more slowly than its corresponding control word when any part of the preceding discourse was consistent with the subordinate meaning of the ambiguous word. That is, the subordinate bias effect emerged when the global context and/or the preceding local context instantiated the subordinate meaning. Previous research (Binder \& Rayner, 1998; Rayner et al., 1994) indicated that initial processing of a biased ambiguous word is strongly influenced by the preceding local context. The present study provided evidence that initial processing of a biased ambiguous word can also be influenced by more distant, global context as long as that context is not changed by local context.

Although global context had an effect in the absence of biasing local context when biasing local context was available prior to encountering the ambiguous target word, global context did not have any additional effect on lexical ambiguity resolution. That is, the magnitude of the subordinate bias effect in the global subordinate-local before condition was the same as in the other two conditions in which an effect was observed (global subordinatelocal after and global dominant-local before). Thus, having two sources of constraint in the preceding context did 
not speed access of the subordinate meaning relative to having one source of constraint. This result is consistent with Rayner et al.'s (1994) second experiment, which also manipulated the amount of subordinately biased contextual information available to the reader prior to encountering a biased ambiguous target word. In their experiment, Rayner et al. found that the subordinate bias effect when the preceding context contained only local information biased toward the subordinate meaning of the ambiguous word was equivalent in magnitude to the subordinate bias effect when the preceding context contained both local and global information biased toward the subordinate meaning. Thus, it seems that when the preceding context establishes a consistent theme, biased toward the subordinate meaning, it does not matter whether that theme was initially established in the global context or established in the global context and continued in the local context. Therefore, maintaining a distinction between local and global context may not be warranted.

The global dominant-local before condition can be seen as a condition that contrasts with the two globalsubordinate conditions because the complete context preceding the ambiguous word does not maintain a consistent theme. The disambiguating information that immediately precedes the ambiguous word in many cases changes the original topic or at least changes the focus from information consistent with the dominant meaning of the ambiguous word to information consistent with the subordinate meaning. Readers were sensitive to this change, as indicated by lengthened reading times on the disambiguating information when it immediately preceded the target word, whether the target was ambiguous or control. The effect of the global-dominant context on meaning resolution for the ambiguous words, however, seems to be slightly delayed, as though the information has been backgrounded to some extent by the shift in focus. This can be seen in the significant effect of ambiguity in the spillover and posttarget region for the global dominantlocal before condition. This may be an indication that the conflict between the subordinate and dominant meanings that begins on the ambiguous word itself spills over into the next region because of a delayed arrival of additional support for the dominant meaning.

Finally, the results for the global dominant-local after conditions are straightforward. When the discourse context contained no information biased toward the subordinate meaning, readers selected the dominant meaning when they first encountered the ambiguous word. Thus there was no difference in initial processing times between the ambiguous target word and the control word. Processing difficulty emerged only when readers encountered the disambiguating information and had to recover from choosing the dominant meaning.

In summary, the results reported here provide support for the claim that global discourse context, as well as local context, immediately influences meaning resolution for ambiguous words. As such, the data are consistent with Hess et al. (1995), who found an effect of global context on the processing of unambiguous words. In addition, our results suggest that global contextual information has no additional effect when it is consistent with local contextual information. However, when the global contextual information is inconsistent with the local contextual information, the lexical ambiguity resolution process is delayed.

\section{REFERENCES}

Binder, K. S., \& Morris, R. K. (1995). Eye movements and lexical ambiguity resolution: Effects of prior encounter and discourse topic. Journal of Experimental Psychology: Learning, Memory, \& Cognition, 21, 1186-1196.

Binder, K. S., \& Rayner, K. (1998). Contextual strength does not modulate the subordinate bias effect: Evidence from eye fixations and self-paced reading. Psychonomic Bulletin \& Review, 5, 271-276.

Binder, K. S., \& RAYNER, K. (1999). Does contextual strength modulate the subordinate bias effect? A reply to Kellas and Vu. Psychonomic Bulletin \& Review, 6, 518-522.

Dopkins, S., Morris, R. K., \& RAYner, K. (1992). Lexical ambiguity and eye fixations in reading: A test of competing models of lexical ambiguity resolution. Journal of Memory \& Language, 31, 461-477.

Duffy, S. A., Kambe, G., \& RaYner, K. (in press). The effect of prior disambiguating context on the comprehension of ambiguous words: Evidence from eye movements. In D. Gorfein (Ed.), On the consequences of meaning selection. Washington, DC: American Psychological Association.

DufFy, S. A., Morris, R. K., \& RAYNER, K. (1988). Lexical ambiguity and fixation times in reading. Journal of Memory \& Language, 27, 429-446.

FRANCIS, W., \& KuČERA, H. (1982). Word frequency counts of modern English. Providence, RI: Brown University Press.

Hess, D. J., Foss, D. J., \& Carroll, P. (1995). Effects of global and local context on lexical processing during language comprehension. Journal of Experimental Psychology: General, 124, 62-82.

IRWIN, D. E. (1998). Lexical processing during saccadic eye movements. Cognitive Psychology, 36, 1-27.

Kellas, G., \& VU, H. (1999). Strength of context does modulate the subordinate bias effect: A reply to Binder and Rayner. Psychonomic Bulletin \& Review, 6, 511-517.

Kintsch, W., \& Mross, E. F. (1985). Context effects in word recognition. Journal of Memory \& Language, 24, 336-349.

Morris, R. K. (1994). Lexical and message-level sentence context effects on fixation times in reading. Journal of Experimental Psychology: Learning, Memory, \& Cognition, 20, 92-103.

Morrison, R. E. (1984). Manipulations of stimulus onset delay in reading: Evidence for parallel programming of saccades. Journal of Experimental Psychology: Human Perception \& Performance, 10, 667-682.

NeILl, W. T. (1989). Lexical ambiguity and context: An activationsuppression model. In D. S. Gorfein (Ed.), Resolving semantic ambiguity (pp. 63-83). New York: Springer-Verlag.

Onifer, W., \& SWInNey, D. A. (1981). Accessing lexical ambiguities during sentence comprehension: Effects of frequency, meaning, and contextual bias. Memory \& Cognition, 9, 225-236.

PACHT, J., \& RAYNER, K. (1993). The processing of homophonic homographs during reading: Evidence from eye movement studies. Journal of Psycholinguistic Research, 22, 251-271.

RAYNER, K. (1998). Eye movements in reading and information processing: Twenty years of research. Psychological Bulletin, 124, 372-422.

RAYNer, K., Binder, K. S., \& Duffy, S. A. (1999). Contextual strength and the subordinate bias effect: Comment on Martin, Vu, Kellas, and Metcalfe. Quarterly Journal of Experimental Psychology, 52A, 841852. 
RAyner, K., \& Duffy, S. A. (1986). Lexical complexity and fixation times in reading: Effects of word frequency, verb complexity, and lexical ambiguity. Memory \& Cognition, 14, 191-201.

Rayner, K., \& Frazier, L. (1989). Selection mechanisms in reading lexically ambiguous words. Journal of Experimental Psychology: Learning, Memory, \& Cognition, 15, 779-790.

Rayner, K., Pacht, J. M., \& Duffy, S. A. (1994). Effects of prior encounter and discourse bias on the processing of lexically ambiguous words: Evidence from eye fixations. Journal of Memory \& Language, 33, 527-544.

Rayner, K., \& Pollatsek, A. (1989). The psychology of reading. Englewood Cliffs, NJ: Prentice-Hall.

Schustack, M. W., Ehrlich, S. F., \& Rayner, K. (1987). Local and global sources of contextual facilitation in reading. Journal of Memory \& Language, 26, 232-252.

SchWANenflugel,P. J., \& White, C. R. (1991). The influence of paragraph information on the processing of upcoming words. Reading Research Quarterly, 26, 160-177.

SEREno, S. C. (1995). The resolution of lexical ambiguity: Evidence from an eye movement paradigm. Journal of Experimental Psychology: Learning, Memory, \& Cognition, 21, 582-595.

Sereno, S. C., Pacht, J. M., \& Rayner, K. (1992). The effect of meaning frequency on processing lexically ambiguous words: Evidence from eye fixations. Psychological Science, 3, 296-300.

Simpson, G. B. (1984). Lexical ambiguity and its role in models of word recognition. Psychological Bulletin, 96, 316-340.

SwinNEY, D. A. (1979). Lexical access during sentence comprehension: (Re)consideration of context effects. Journal of Verbal Learning \& Verbal Behavior, 18, 645-659.

Tanenhaus, M. K., Leiman, J. M., \& Seidenberg, M. S. (1979). Evidence for multiple stages in the processing of ambiguous words in syntactic contexts. Journal of Verbal Learning \& Verbal Behavior, 18, 427-440.

Till, R. E., Mross, E. F., \& Kintsch, W. (1988). Time course of priming for associate and inference words in a discourse context. Memory \& Cognition, 16, 283-298

Twilley, L. C., Dixon, P., Taylor, D., \& Clark, K. (1994). University of Alberta norms of relative meaning frequency for 566 homographs. Memory \& Cognition, 22, 111-126.

Vu, H., \& Kellas, G. (1999). Contextual strength modulates the subordinate bias effect: Reply to Rayner, Binder, and Duffy. Quarterly Journal of Experimental Psychology, 52A, 853-856.

Wiley, J., \& RAYNER, K. (2000). Effects of titles on the processing of text and lexically ambiguous words: Evidence from eye movements. Memory \& Cognition, 28, 1011-1021.

\section{NOTES}

1. There is some controversy over whether or not the subordinate bias effect can be eliminated by strong context and the implications for the reordered access model (see Binder \& Rayner, 1999; Kellas \& Vu, 1999; Rayner, Binder, \& Duffy, 1999; Vu \& Kellas, 1999). Indeed, Wiley and Rayner (2000) recently found that strong contexts can result in the earlier access of the subordinate meaning of biased words for all but very infrequent subordinate meanings. As noted by Rayner et al (1999), the reordered access model can handle override of the subordinate bias effect via strong contextual constraints. However, that issue is beyond the scope of the present paper.

2. There is some question (see Pacht \& Rayner, 1993; Rayner \& Duffy, 1986; Sereno et al., 1992) as to whether or not control words for ambiguous words should be matched on overall word frequency or meaning frequency. A meaning frequency match for the subordinate meaning of a biased ambiguous word would mean that if the overall frequency of an ambiguous word was 100 , but the subordinate meaning was selected only $5 \%$ of the time, the appropriate control word should have a frequency of 5 . In the present experiment, we used overall word frequency matches because the most important contrasts in the data can be most straightforwardly examined by comparing gaze durations on the ambiguous word with gaze durations on the overall frequencymatched control word. A difference in these two measures indicates that context has had an immediate effect on processing of the ambiguous word, and this is the focus of the current work. See Duffy, Kambe, and Rayner (in press) for an extended discussion of this issue.

3. As we have argued elsewhere (Duffy et al., 1988; Rayner et al., 1994), standard exhaustive or selective access models cannot account for the pattern of eye movement data typically obtained. Thus, some type of hybrid model is necessary in which meaning frequency and contextual information influence ambiguity resolution. The predictions made here are presented within the context of the reordered access model.

4. An analysis in which saccade durations were added to the gaze duration (Irwin, 1998) yielded exactly the same results as reported here. The inclusion of saccade durations simply added a couple of milliseconds to those in Table 1 and tended to make the difference between the ambiguous and control words slightly larger (though not for the global dominant-local after condition).

5. ANOVAs on the first fixation data and total time revealed the same effects: first fixation duration $\left[F_{1}(1,47)=8.01, M S_{\mathrm{e}}=1,421, F_{2}(1,47)=\right.$ $\left.6.7, M S_{\mathrm{e}}=2,195\right]$; total time $\left[F_{1}(1,47)=10.21, M S_{\mathrm{e}}=6,680, F_{2}(1,47)\right.$ $\left.=9.59, M S_{\mathrm{e}}=9,487\right]$. Likewise, in these analyses there were no differences between the control and target word for the global dominant-local after condition $(F \mathrm{~s}<1)$.

6 . Reading times on the control word were a bit longer in the global dominant-local after condition than in the other control conditions. This difference was not significant (all $F \mathrm{~s}<1$ ). Because the control words have only one meaning, a decision was necessary as to which context (the subordinate or dominant) would be most appropriate to have the control word fit into. A completely neutral word (such as soup in the example passages in Appendix A) would have been anomalous with the entire discourse. The control word was selected to be consistent with the local or subordinately biased contexts. As a result, when dominantly biased context preceded the control word with no preceding subordinately biased contextual information, the control word was not as good a fit into the discourse. This occurred only in one passage condition, and while perhaps noticeable to the reader, was very infrequent (occurring only eight times in a total of 96 passages).

\section{APPENDIX A \\ Example Stimuli (Target Words in Italics)}

\section{Version 1}

\section{Global Subordinate-Local After}

Lisa and John spent months looking for the perfect wedding ring. They had a great time traveling around together. They were especially pleased that they both liked the same kinds of things. One day they decided to go to New York City to see the band/gold that would be made into an exact duplicate of a ring worn by Cleopatra.

\section{Global Subordinate-Local Before}

Lisa and John spent months looking for the perfect wedding ring. They had a great time traveling around together. They were especially pleased that they both liked the same kinds of things. It wasn't until they entered Kay's Jewelry store that they saw the band/gold that would make the perfect wedding ring for both of them.

\section{Global Dominant-Local After}

Lisa and John loved to go to rock concerts with their friends. They had a great time traveling around together. They were especially pleased that they both liked the same kinds of things. One day they decided to go to New York City to see the band/ gold that would be made into an exact duplicate of a ring worn by Cleopatra. 


\section{APPENDIX A (Continued)}

\section{Global Dominant-Local Before}

Lisa and John loved to go to rock concerts with their friends. They had a great time traveling around together. They were especially pleased that they both liked the same kinds of things. It wasn't until they entered Kay's Jewelry store that they saw the band/ gold that would make the perfect wedding ring for both of them.

\section{Version 2}

\section{Global Subordinate-Local After}

Mary Jo was surprised that the actor was wearing a wedding ring. She had been at the interview for fifteen minutes. Everything was going well and she was very pleased. The conversation continued to flow and eventually turned to the band/gold that he had engraved by a jeweler as surprise for his wife.

\section{Global Subordinate-Local Before}

Mary Jo was surprised that the actor was wearing a wedding ring. She had been at the interview for fifteen minutes. Everything was going well and she was very pleased. She could not help but notice the elaborate engraving that decorated the band/ gold that he had on his ring finger.

\section{Global Dominant-Local After}

Mary Jo could not believe that she got to meet Bono from U2. She had been at the interview for fifteen minutes. Everything was going well and she was very pleased. The conversation continued to flow and eventually turned to the band/gold that he had engraved by a jeweler as surprise for his wife.

\section{Global Dominant-Local Before}

Mary Jo could not believe that she got to meet Bono from U2. She had been at the interview for fifteen minutes. Everything was going well and she was very pleased. She could not help but notice the elaborate engraving that decorated the band/gold that he had on his ring finger.
APPENDIX B

List of All Ambiguous Target and Control Words (Matched on Length and Frequency)

\begin{tabular}{|c|c|}
\hline Ambiguous Target Words & Control Words \\
\hline cabinet & analyst \\
\hline port & beer \\
\hline bank & edge \\
\hline notes & songs \\
\hline boxer & puppy \\
\hline speaker & machine \\
\hline pipes & drain \\
\hline poker & sword \\
\hline band & gold \\
\hline ball & test \\
\hline coach & cabin \\
\hline scale & stone \\
\hline wire & card \\
\hline horn & tail \\
\hline racket & tenant \\
\hline diamond & parkway \\
\hline table & paper \\
\hline legend & harbor \\
\hline story & floor \\
\hline plant & hotel \\
\hline corn & wart \\
\hline pen & ZOO \\
\hline habit & cross \\
\hline $\operatorname{mint}$ & jail \\
\hline
\end{tabular}

Note-All ambiguous words are noun-noun ambiguities.

(Manuscript received January 26, 2000; revision accepted for publication July 12, 2000.) 